EESTI NSV TEADUSTE AKADEEMIA TOIMETISED. 31. KOIDE

FOOSIKA * MATEMAATIKA. 1982, NR. 3

ИЗВЕСТИЯ АКАДЕМИИ НАУК ЭСТОНСКОИ ССР. ТОМ 31 ФИЗИКА * МАТЕМАТИКА. 1982, № 3

УдК $681.2 .081: 53$

И. НОВИКОВ, А. ПОЛНАРЕВ, И. РОЗЕНТАЛЬ

\title{
ЧИСЛЕННЫЕ ЗНАЧЕНИЯ ФУНДАМЕНТАЛЬНЫХ ПОСТОЯННЫХ И АНТРОПНЫЙ ПРИНЦИП *
}

\author{
(Представил К. К. Ребане)
}

Целью данной работы является дальнейшее развитие идей антропного принципа $\left[{ }^{1-4}\right]$ (или, иначе, принципа целесообразности - термин одного из авторов настоящей статьи $\left.\left[{ }^{5}\right]\right)$. Основная идея антропного принципа состоит в том, что фундаментальные свойства Вселенной, значения основных физических констант и даже форма физических закономерностей тесно связаны с фактом структурности Вселенной во всех масштабах от элементарных частиц до сверхскоплений галактик, с возможностью существования условий, при которых возникают сложные формы движения материи и, в конце концов, жизнь и человек.

Проблема возникновения структурности и жизни во Вселенной традиционно трактуется следующим образом. Окружающая нас Вселенная обладает определенными физическими свойствами и закономерностями, познаваемыми нами. Как в таком случае происходит эволюция Вселенной, приводящая к достаточно сложным структурам, как зарождается и эволюционирует в такой Вселенной жизнь? От ответа на эти, во многом еще не решенные, вопросы зависят ответы на такие вопросы, как возможна ли жизнь в других областях Вселенной и в другие времена и как ее искать.

Антропный принцип позволяет подойти к проблеме совершенно иначе. Прежде чем мы перейдем к обсуждению этого подхода, который в настоящее время широко дискутируется в научной литературе, сделаем следующее замечание. Любая физическая теория, например уравнения Максвелла в электродинамике, ставит перед собой задачу дать полное физическое описание той или иной системы, если известен полный набор начальных данных и, разумеется, фундаментальных констант теории. Перед теорией, как правило, не стоит задача объяснения начальных данных, поскольку в различных физических явлениях начальные данные различны. Но когда мы обращаемся к космологии, которая должна описать свойства одной единственной системы - нашей Вселенной, вопрос о начальных данных и фундаментальных постоянных неразрывно связан с вопросом, почему Вселенная именно такая, какой мы ее наблюдаем $\left[{ }^{6-8}\right]$.

Суть антропного принципа заключается в следующем: Вселенная такова, какой мы ее видим, потому что в ней существуем мы, т. е. наблюдатели, способные задать вопрос о свойствах Вселенной; при других параметрах во Вселенной невозможны сложные структуры и жизнь в известных нам формах.

Более обобщенно аналогичный вопрос можно сформулировать следующим образом (принцип целесообразности): ни в какой другой Вселенной (если вообразить, что где-то или когда-то возможны другие Вселенные, или хотя бы представить множество «виртуальных» - в

* Работа доложена на Международном симпозиуме «Поиск разумной жизни во Вселенной» (Таллин, 7-12 декабря 1981 г.). 
принципе, возможных Вселенных) со слегка измененными фундаментальными постоянными или с другими законами физики невозможно образование сложных устойчивых структурных единиц, атомов, молекул, планет, звезд, галактик и, разумеется, существование высокоорганизованной органической материи.

На первый взгляд это утверждение кажется странным. Действительно, если мы изменим, например, заряд электрона в несколько раз или изменим его массу, то соответственно изменится размер атомов и в соответствующее число раз изменится лишь масштаб всех предметов. Представляется, что то же самое произойдет и с другими закономерностями: скажем, увеличим гравитационную постоянную, тогда изменятся размеры небесных тел, изменятся сроки эволюции звезд, но, в принципе, и в этом случае в такой вселенной будут существовать атомы, небесные тела, сложные химические структуры и, возможно, жизнь. Оказывается это не так. Как показано в $\left[{ }^{5,9-11}\right]$, даже небольшие изменения фундаментальных постоянных приводят к качественным изменениям свойств Вселенной, в частности, к невозможности существования сложных структур, а значит, и жизни (см. $\left.\left[{ }^{10}\right]\right)$.

Однако до сих пор оставался открытым вопрос - а нельзя ли согласованно и сильно изменить весь набор физических констант, параметров Вселенной (а в принципе, и физических законов) так, чтобы получить модели других вселенных, в которых выполнены если не достаточные, то хотя бы необходимые условия для возникновения сложных структур и жизни?

Таким образом, возникает проблема - исследовать возможные процессы (всех масштабов!) и эволюцию Вселенной, используя совсем иную физику. Во всем объеме такая задача сегодня не разрешима. Можно ограничиться более узкой задачей - исследовать свойства Вселенной при всевозможных значениях какой-нибудь группы констант. Если откладывать значения этих констант на осях в некотором фазовом пространстве, то можно попытаться выделить в этом пространстве область такую, что если значения констант принадлежат этой области, то во Вселенной существуют сложные структуры. Эту область можно назвать «островом устойчивости структур». Сформулированная выше задача соответствует попытке отыскания других «островов устойчивости» при сильно измененных значениях констант. В данной работе мы попытаемся это сделать.

Как отмечено в $\left[{ }^{3}\right]$, основные масштабы масс, длин и времен во Bселенной определяются следующими тремя безразмерными фундаментальными постоянными:

1) постоянной тонкой структуры

$$
\alpha_{e}=e^{2} / \hbar c \text {, }
$$

где $e-$ заряд электрона, $\hbar$ - постоянная Планка и $c-$ скорость света;

2) безразмерной гравитационной постоянной

$$
\alpha_{g}=G m_{p}^{2} / \hbar c
$$

где $G$ - ньютоновская постоянная тяготения, а $m_{p}$ - масса протона;

3) отношением массы электрона к массе протона $m_{e} / m_{p}$. Это отношение связано, как показано в $\left[{ }^{3}\right]$, с величиной $\alpha_{e}$ следующим соотношением

$$
m_{e} / m_{p} \sim 10 \alpha_{e}^{2} .
$$

Соотношение (3) вытекает из следующих требований, необходимых для существования элементов, которые, в свою очередь, необходимы для возникновения жизни. 
Во-первых, сйльные взаимодействия не могут быть нй существённо слабее, иначе нуклоны не могли бы связываться в ядра и из всех элементов существовал бы только водород, ни существенно сильнее, в противном случае масса ядер могла бы быть сколь угодно большой и не было бы ни одного устойчивого ядра с конечной массой. Во-вторых, нейтроны должны быть нестабильны по отношению к $\beta$-распаду, если они свободны и устойчивы к $\beta$-распаду в связанном состоянии в ядрах. В-третьих, электростатическая энергия в легких ядрах должна быть сравнима с разницей масс между нейтроном и протоном. И, в-четвертых, электростатическая энергия в легких ядрах должна быть мала по сравнению с энергией связи в них, но сопоставима с энергией связи в тяжелых ядрах, т. е. должна быть такой, чтобы очень тяжелые ядра были неустойчивы относительно распада при воздействии электромагнитного поля.

Мы здесь не будем обсуждать, что произойдет при изменении других констант, например безразмерной энтропии, т. е. числа фотонов, приходящихся на один барион, или безразмерной константы слабого взаимодействия (вновь отсылаем читателя к цитированным выше работам).

В данной работе мы ограничимся рассмотрением всех возможных взаимосвязанных вариаций двух основных констант $\alpha_{e}$ и $\alpha_{g}$. Для этого остановимся только на тех необходимых для существования сложных структур условиях, которые не зазисят от других констант, а зависят только от $\alpha_{e}$ и $\alpha_{g}$.

Принципиальная возможность существования сложных элементов, обязательных для возникновения жизни, как уже говорилось, обеспечивается выполнением условия (3). Но для синтеза элементов необходимо существование звезд. Кроме того, для планетных систем необходимы, как отмечается в [ $\left.{ }^{1}\right]$, конвективные звезды. Там же показано, что для этого должно выполняться неравенство

$$
\alpha_{g} \geqslant \alpha_{e}^{12}\left(m_{e} / m_{p}\right)^{4},
$$

которое с учетом (3) принимает вид

$$
\alpha_{g} \geqslant \alpha_{e}^{20} \text {. }
$$

Для образования звезд, по-видимому, необходимо существование галактик. Галактики же могут сформироваться лишь после того, как произойдет процесс рекомбинацин водорода в расширяющейся Вселенной. Для этого температура во Вселенной должна упасть ниже, чем

$$
k T \sim 0,1 \alpha_{e}^{2} m_{e} c^{2} .
$$

Необходимо отметить, что для возникновения небесных тел Вселенная не должна быть «слишком открытой», т. е. плотность в ней не должна быть сегодня существенно меньше критической. Иначе в ней не происходил бы достаточный рост начальных возмущений плотности за счет гравитационной неустойчивости. С другой стороны, Вселенная не может быть «слишком замкнутой», потому что полное время ее расширения было бы слишком мало и в ней не успевало бы происходить горение водорода в звездах, приводящее к образованию тяжелых элементов. Эти элементы, вероятно, необходимы для появления сложных химических соединений и жизни. То есть плотность во Вселенной должна быть близка к критической в эпохи, сравнимые со временем эволюции звезд. Кроме того, это последнее условие означает, что эпоха доминантности вещества над излучением не может начаться задолго до эпохи рекомбинации. На стадии доминирования излучения температура зависит от времени по закону $\left[{ }^{12}\right]$ : 


$$
k T \simeq\left(\hbar^{3} c^{5} / G t^{2}\right)^{1 / 4}
$$

Потребовав, чтобы время, когда происходит рекомбинация, было меньше характерного времени жизни звезд на главной последовательности $\left[{ }^{3}\right]$

$$
t_{\mu s} \sim \pi \alpha_{g}^{-1} / m_{p} c^{2},
$$

с учетом (3), (6)-(8), а также при условии, что плотность Вселенной близка к критической, получаем

$$
\alpha_{g}<\alpha_{e}^{16}
$$

Еще одно ограничение на $\alpha_{e}$ и $\alpha_{g}$ можно получить, основываясь на гипотезе о возможности объединения всех физических взаимодействий в рамках единой теории $\left[{ }^{13}\right]$. Потребовав, чтобы характерная масса такого объединения, необходимая для устранения расходимостей в квантовой электродинамике, была меньше планковской, имеем

$$
\alpha_{g}<e^{-1 / 2 \alpha_{e}} .
$$

Можно возразить тому, что условие (10) вытекает из антропного принципа. Действительно, здесь скорее можно говорить о принципе целесообразности. Мы думаем, нто в реальности нет расходимостей, с которыми сталкивается теория квантовой электродинамики, а выполнение условия (10) - это самый естественный путь устранения этих расходимостей в будущей единой теории взаимодействий.

Кроме того, теория объединения слабого, электромагнитного и сильного взаимодействий предсказывает нестабильность протона относительно распада на лептоны.

Постулируя квазистабильность протона, т. е. требуя, чтобы протон был практически стабилен в течение времени, необходимого для возникновения сложных структур - галактик, звезд и т. д., получаем неравенство $\left[{ }^{13}\right]$

$$
\alpha_{g}>\alpha_{e}^{2} e^{-1 / 2 \alpha_{e}}
$$

Разумеется, во всех неравенствах мы пренебрегали, где это возможно, величинами порядка единицы.

Совместное решение неравенств (5), (9)-(11) позволяет найти на плоскости параметров $\left(\alpha_{e}, \alpha_{g}\right)$ области допустимых значений, где выполняются необходимые (хотя, сразу подчеркнем, отнюдь не достаточные) условия для возникновения сложных устойчивых структур. Следует подчеркнуть, что учет дополнительных ограничений может лишь сузить разрешенную область.

Найденные в результате решений системы (5), (9)-(11) области будут «островами стабильности структур» в том смысле, как мы указывали выше. При формальном решении мы неограниченно экстраполируем полученные неравенства заведомо далеко за пределы их доказанной справедливости. Тем не менее мы идем на такую чисто формальную экстраполяцию с методической целью пояснить нашу основную мысль как искать модели других вселенных, в которых возможны сложные структуры.

Введем следующие переменные, с которыми удобно работать:

$$
x=\log \alpha_{e}^{-1}, \quad y=\log \alpha_{g}^{-1 / 4} .
$$

Тогда система неравенств (5), (9)-(11) принимает вид

$$
\begin{aligned}
& y<5 x \\
& y>4 x,
\end{aligned}
$$




$$
\begin{aligned}
& y>\frac{1}{8} \log e \cdot 10^{x} \\
& y<\frac{x}{2}+\frac{\log e}{4} \cdot 10^{x}
\end{aligned}
$$

На рисунке показаны разрешенные области параметров - «острова устойчивости структур»:

\begin{tabular}{ll|l|l|l|l|l|l|l|r}
\hline & $A$ & $B$ & $C$ & $D$ & $A^{\prime}$ & $B$ & $C^{*}$ & $D^{\prime}$ & $O$ \\
\hline$X$ & 1 & 2 & 2 & 1 & 0,01 & 0,01 & 0,2 & 0,1 & 2 \\
$\mathrm{y}$ & 3 & 9 & 12 & 5 & 0,1 & 0,1 & 1 & 1 & 10
\end{tabular}

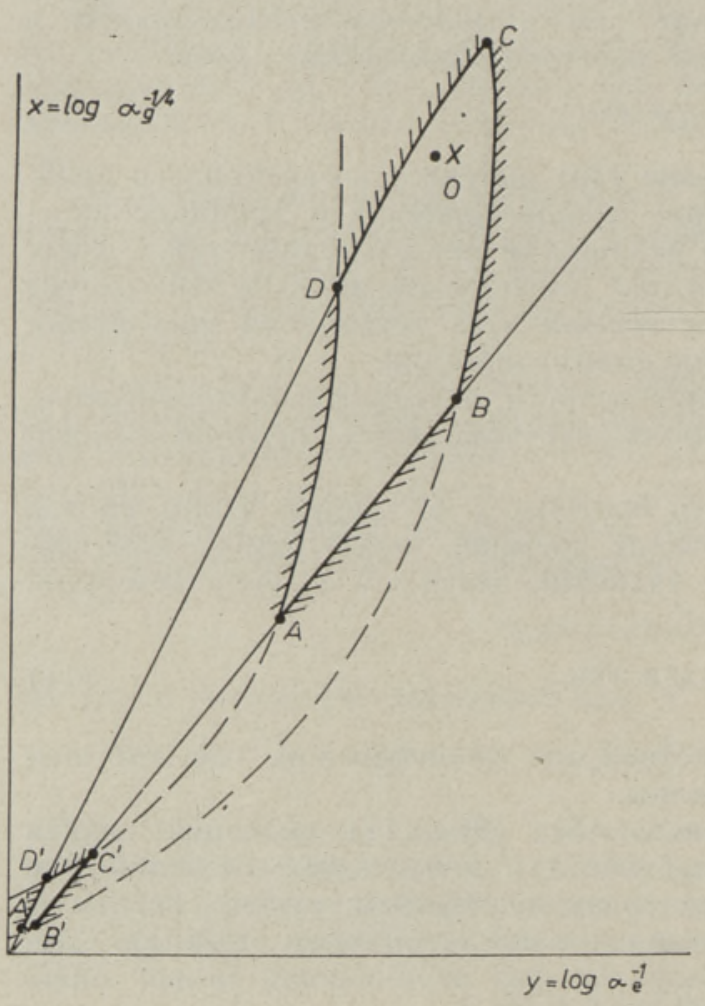

Области, где выполняется ряд необходимых условий для возникновения жизни (точка 0 соответствует нашей Вселенной).

Оказалось, что имеются две отдельные области: область $A B C D$, внутрь которой попадает наша Вселенная

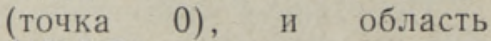
$A^{\prime} B^{\prime} C^{\prime} D^{\prime}$, , которая соответствует $x \ll 1, y<1$, т. $\mathrm{e}$. соответствует семейству вселенных, в которых $\alpha_{e}$ и $\alpha_{g}$ близки к единице. Ясно, что в таких вселенных все характерные времена, размеры и массы близки к соответствующим планковским значениям. Поэтому, разумеется, в таких вселенных очень сложные структуры и жизнь невозможны: слишком мало объектов (масга каждого объекта порядка массы вселенной, порядка планковской массы).

Итак, если исходить из известных закономерностей и из полученных при «наивной» неограниченной экстраполяции необходимых неравенств, то можно прийти к заключению: существует, вероятно, только одна область параметров $\alpha_{e}$ и $\alpha_{g}$, при которых возможно возникновение сложных структур и жизни во Вселенной. Подчеркнем, что область $A B C D$ - это область, где выполняются необходимые условия для возникновения сложных структур. Область же необходимых и достаточных условий может быть существенно у́же.

Конечно, детальное изучение этого вопроса может привести к иным математическим соотношениям, чем использованная нами система неравенств. Эти другие соотношения могут сильно отличаться от наших при значениях $\alpha_{g}$ и $\alpha_{e}$ существенно иных, чем наблюдаемые нами. Тогда, возможно, появятся и другие «острова устойчивэсти». 
В заключе́ние еще раз подцеркнем, что целью настоящей работы являлась постановка вопроса о поисках моделей вселенных с параметрами, сильно отличающимися от параметров нашей Вселенной, но при которых, тем не менее, возможны сложные структуры.

\section{ЛИ Т Е РА Т У Р А}

1. К а р тер Б., В кн.: Космология. Теории и наблюдения, М., «Мир», 1978.

2. Misner, C. W., Thorne, K. S., Wheeler, J. A., Gravitation, Freeman, San Francisco, 1973; (русск. перевод: Мизнер Ч. В., Торн К. С., Уилер Дж. А., Гравитация, М., «Мир», 1977).

3. C a r r, B. J., R e e s, M. J., Nature, 2:8, № 5705, 605-612 (1979).

4. З е л ьд ов и ч Я. Б., Письма в АЖ, 7, № 10, 579-581 (1981).

5. Р оз е н т а ль И. Л., Успехи физ. наук, 121, 239-256 (1980).

6. D i r a c, P. A. M., Nature, 139,323 (1937).

7. И д ли с Г. М., Изв. Астроф. Ин-та КазССР, 7, 38 (1958).

8. Di c k e, R. H., Nature, 192, 440 (1961).

9. Р оз ент а ль И. Л., Пр-400, ИКИ АН СССР, 1978.

10. Розен таль И. Л., Пр-608, ИКИ АН СССР, 1980.

11. Р озен т аль И. Л., Пр-636, ИКИ АН СССР, 1981.

12. Зельдович Я. Б., Новиков И. Д., Строение и эволюция Вселенной, М., «Наука», 1975.

13. Р озенталь И. Л., Письма в ЖЭТФ, 31, вып. 9, 521-524 (1980).

Ннститут космических исследований Академии наук СССР
Поступила в редакцию $24 /$ II 1982

\section{NOVIKOV, A. POLNARJOV, I. ROZENTAL}

\section{FUNDAMENTAALKONSTANTIDE ARVVÄÄRTUSED JA ANTROOPSUSPRINTSIIP}

On analüüsitud füüsika fundamentaalkonstantide arvväärtuse probleemi ning näidatud, et niisugused universumi mudelid, mil!es konstantide väärtused erinevad oluliselt tegelikult vaadeldavatest väärtustest, välistavad keeruliste struktuuride (ja elu) olemasolu (universumi struktuuri lokaalse püsivuse printsiip looduse konstantide variatsioonide suhtes). On formuleeritud niisuguse universumi mudeli otsimise probleem, milles füüsikaliste konstantide väärtused väga palju erinevad nende väärtustest meie universumis (lokaalse püsivuse teiste piirkondade otsimine), ning esitatud sellise otsingu näide.

\section{NOVIKOV, A. POLNARYOV, I. ROZENTAL}

\section{ON NUMERICAL VALUES OF FUNDAMENTAL CONSTANTS AND THE PRINCIPLE OF ANTROPY}

The problem of numerical values of fundamental physical constants is analysed. Impossibility of existence of complicated structures - including Life - in models of the Universe with the values of the constants significantly different from the observed values is pointed out (the principle of local stability of the structure of the Universe with respect to variations of the constants of Nature). The problem of searching for much models of the Universe where the values of the physical constants would greatly differ from those in our Universe is formulated - as a search for different areas of local stability. An example of such a search is presented. 